\title{
The Weak Temperature Gradient Approximation and Balanced Tropical Moisture Waves*
}

\author{
AdAm H. Sobel \\ Department of Applied Physics and Applied Mathematics, and Department of Earth and Environmental Sciences, Columbia University, \\ New York, New York \\ JOHAN NILSSON \\ Department of Meteorology, Stockholm University, Stockholm, Sweden \\ LORENZO M. POLVANI \\ Department of Applied Physics and Applied Mathematics, and Department of Earth and Environmental Sciences, Columbia University, \\ New York, New York
}

(Manuscript received 14 December 2000, in final form 30 May 2001)

\begin{abstract}
Horizontal temperature gradients are small in the tropical atmosphere, as a consequence of the smallness of the Coriolis parameter near the equator. This provides a strong constraint on both large-scale fluid dynamics and diabatic processes. This work is a step toward the construction of a balanced dynamical theory for the tropical circulation that is based on this constraint, and in which the diabatic processes are explicit and interactive.

The authors first derive the basic fluid-dynamical scaling under the weak temperature gradient (WTG) approximation in a shallow water system with a fixed mass source representing an externally imposed heating. This derivation follows an earlier similar one by Held and Hoskins, but extends the analysis to the nonlinear case (though on an $f$ plane), examines the resulting system in more detail, and presents a solution for an axisymmetric "top-hat" forcing. The system is truly balanced, having no gravity waves, but is different from other balance models in that the heating is included a priori in the scaling.

The WTG scaling is then applied to a linear moist model in which the convective heating is controlled by a moisture variable that is advected by the flow. This moist model is derived from the Quasi-equilibrium Tropical Circulation Model (QTCM) equations of Neelin and Zeng but can be viewed as somewhat more general. A number of additional approximations are made in order to consider balanced dynamical modes, apparently not studied previously, which owe their existence to interactions of the moisture and flow fields. A particularly interesting mode arises on an $f$ plane with a constant background moisture gradient. In the limit of low frequency and zero meridional wavenumber this mode has a dispersion relation mathematically identical to that of a barotropic Rossby wave, though the phase speed is eastward (for moisture decreasing poleward in the background state) and the propagation mechanism is quite different. This mode also has significant positive growth rate for low wavenumbers. The addition of the $\beta$ effect complicates matters. For typical parameters, when $\beta$ is included the direction of phase propagation is ambiguous, and the growth rate reduced, as the effects of the background gradients in moisture and planetary vorticity appear to cancel to a large degree. Possible relevance to intraseasonal variability and easterly wave dynamics is briefly discussed.
\end{abstract}

\section{Introduction \\ a. Background}

Horizontal density and temperature gradients are very small in the Tropics. The well-known consequence of

\footnotetext{
*Lamont-Doherty Earth Observatory Contribution Number LDEO 6190.

Corresponding author address: Dr. Adam H. Sobel, Department of Applied Physics and Applied Mathematics, Columbia University, 500 West 120th St., Rm. 217, New York, NY 10027.

E-mail: sobel@appmath.columbia.edu
}

this is that, on large scales, the dominant balance in the temperature equation is between heating and vertical advection of potential temperature. A tropical heat source causes vertical motion and an associated horizontal flow but only small temperature changes. A number of studies have neglected horizontal temperature variations as a simplifying assumption in idealized models of the tropical climate (e.g., Neelin and Held 1987; Pierrehumbert 1995; Miller 1997; Zeng 1998; Zeng and Neelin 1999; Raymond 2000; Clement and Seager 1999; Sobel and Bretherton 2000, hereafter SB00). SB00 suggest that this assumption may be able to serve as the 
foundation of balanced dynamical models for the study of tropical dynamics that explicitly include diabatic processes. In such models, both the "dry" aspects of the fluid dynamics and the diabatic processes would be constrained by the assumption of nearly horizontally uniform free tropospheric temperature. Radiative transfer becomes governed essentially by tropospheric humidity, cloudiness, and surface properties when the free tropospheric temperature profile is held fixed. Convection becomes governed by near-surface temperature and humidity ( since these largely control the convective available potential energy and convective inhibition), and also by free tropospheric relative humidity, since this governs the effectiveness with which entrainment of environmental air into clouds can suppress convection. In such models, then, moisture becomes an important dynamical variable. The humidity of the atmosphere at a given horizontal location-leaving aside for the moment questions about vertical structure-can have a strong effect on the diabatic heating at that location and, hence, on the vertical velocity and horizontal divergence. In the presence of background absolute vorticity, this divergence in turn drives a large-scale rotational flow through vortex stretching and horizontal advection of absolute vorticity.

Though atmospheric water vapor is not conserved in the presence of precipitation and surface evaporation, the horizontal advection of water vapor does have an influence on its large-scale horizontal distribution and thus on the distribution of deep convection. Chen et al. (1996) noted that the occurrence of deep convection during the Tropical Ocean and Global Atmosphere Coupled Ocean-Atmosphere Response Experiment (TOGA COARE) field experiment appeared to be linked to the direction of the low-level zonal wind more than to its speed. The surface wind speed would control convection if surface fluxes were the factor of primary importance, as in some quasi-equilibrium theories (e.g., Emanuel et al. 1994, and references therein). On the other hand, Chen et al. (1996) suggested that in the TOGA COARE intensive flux array, westerlies carry relatively moist air compared to easterlies, so that the asymmetry could be partly explainable through the advection of water vapor. Spectacular examples of the same process came in the same experiment when the advection of very dry subtropical air from the north was observed to suppress convection (Parsons et al. 1994; Numaguti et al. 1995; Yoneyama and Fujitani 1995; Yoneyama and Parsons 1999). Evidence of a more general, statistical nature for the dynamical importance of free tropospheric humidity was presented by Sherwood (1999). From a modeling perspective, the importance of horizontal water vapor advection is shown explicitly in the simulations of SB00 using modified versions of the models of Neelin and Zeng (2000, hereafter NZ00; see also Zeng et al. 2000) and Rennó et al. (1994a,b).

\section{b. Outline}

In this study, we first elucidate (section 2a) the basic scaling under what we call, for obvious reasons, the weak temperature gradient (WTG) approximation. In this approximation, horizontal temperature gradients are neglected at leading order. Small temperature perturbations can be obtained at next order from a balance requirement on the leading order flow. For maximum simplicity, the scaling analysis is performed using a shallow water system driven by a given mass source, analogous to a heating. The basic dynamics of this model has, in essence, been presented by Held and Hoskins (1985; hereafter HH85); similar ideas can also be found in the discussions of Sardeshmukh and Hoskins (1987, 1988). Compared to HH85, we formulate the problem somewhat differently, allow for nonlinearity, present an explicit solution with emphasis on the local tropical response (as opposed to teleconnections to midlatitudes), and address some additional details.

The purpose of the rest of the paper is to illustrate some types of large-scale dynamical behavior, which we hypothesize can result from horizontal moisture advection in the Tropics. In order to understand these effects as clearly as possible we use a model framework with a number of strong simplifications. The first set of simplifications can be viewed as being embodied in the single-mode vertical structure and simple convective parameterization, as in the derivation of the Quasi-equilibrium Tropical Circulation Model (QTCM) developed at the University of California, Los Angeles (Neelin and Yu 1994; Yu and Neelin 1994; Neelin 1997; Neelin and Zeng 2000; Zeng et al. 2000), which we take as our starting point. We then make a number of further assumptions, perhaps the most restrictive of which is the use of an $f$ plane to eliminate Rossby waves. This is of course unrealistic, but it is done in order to isolate the dynamics of interest. In particular, in the presence of a background horizontal gradient of moisture, modes exist that are somewhat reminiscent of Rossby waves. Use of an $f$ plane brings these modes into better relief. The addition of the $\beta$ effect is considered briefly, but a thorough analysis of its role in this system is deferred.

\section{Weak temperature gradient scaling in the shallow water system}

a. Scaling

Consider the dimensional shallow water equations on an $f$ plane, subject to an imposed mass source and Rayleigh friction:

$$
\begin{aligned}
\frac{\partial h}{\partial t}+\boldsymbol{\nabla} \cdot(\mathbf{u} h) & =Q \\
\frac{\partial \zeta}{\partial t}+\boldsymbol{\nabla} \cdot[\mathbf{u}(\zeta+f)] & =-\alpha \zeta \\
\frac{\partial \delta}{\partial t}+\frac{1}{2} \nabla^{2}(\mathbf{u} \cdot \mathbf{u})+g \nabla^{2} h-\hat{\mathbf{k}} \cdot \boldsymbol{\nabla} \times[\mathbf{u}(\zeta+f)] & =-\alpha \delta .
\end{aligned}
$$


Fluid depth, velocity, vorticity, and divergence are denoted as $h, \mathbf{u}, \zeta$, and $\delta$, respectively, mass source is $Q$; dissipation rate $\alpha$; gravity and coriolis parameters are $g$, and $f$, respectively; and $\hat{\mathbf{k}}$ is the vertical unit vector. We separate the fluid depth into a constant mean and fluctuating perturbation, $h=H+\eta(\mathbf{x}, t)$, where $\mathbf{x}$ denotes horizontal position.

The shallow water system can describe, with proper interpretation of the variables, the dynamics of a stratified compressible atmosphere, if the vertical structure of the motion is taken fixed. The most common set of assumptions used for this is that of linear normal mode theory (e.g., Matsuno 1966; Lindzen 1967; Gill 1980; among others), but somewhat different approximations can lead to a fundamentally similar result, as in the QTCM equations. Due to the multiplicity of interpretations, we use a "raw" shallow water system in this section to illustrate the fundamental scaling.

\section{BASIC SCALING ASSUMPTION}

We are interested in situations, corresponding to weak temperature gradients, where the leading order balance in (1) is

$$
H \delta \sim Q
$$

consistent with small temperature gradients. Given the externally imposed scales $L, T, Q_{0},{ }^{1}$ which characterize the forcing, and $H$ and $f$, which characterize the basic state, we wish to establish under which conditions this leading order balance is attained. A logical choice of velocity scale is

$$
U \sim Q_{0} L / H .
$$

Also, to begin with we assume that

$$
\zeta \sim \delta \sim Q_{0} / H
$$

Equation (5) states that the divergent and rotational components of the flow have equal magnitude. This assumption will be relaxed in the linear limit (below) but is used as a starting point to emphasize that our scaling differs fundamentally from quasi-balanced adiabatic dynamics, whether near or far from the equator. In fact the rotational flow in the real tropical troposphere is typically larger than the divergent flow (on large scales) but not by an order of magnitude.

What remains is to identify an appropriate scale for $\eta$. Anticipating that rotation influences the dynamics, the scale of $\eta$ can be chosen from (3):

\footnotetext{
${ }^{1}$ Note that we assume only a single length scale, rather than two distinct scales, for the meridional and zonal directions. This is consistent with the fact that most calculations to follow will not explicitly include the $\beta$ effect. However, our basic scaling analysis holds on a beta plane (and becomes very similar to that of HH85) if we take $f$ $\sim \beta L$. Our length scale must be defined by the length scale of $Q$, so for the present analysis we assume $Q$ is not strongly asymmetric in $x$ and $y$.
}

$$
g \nabla^{2} h \sim \hat{\mathbf{k}} \cdot \nabla \times(\mathbf{u} f)
$$

this is not necessarily the dominant balance, it just states that the terms are of the same order. This yields

$$
\eta_{0} \sim Q_{0} f L^{2} /(g H)
$$

Using these scales to put the shallow water equations in nondimensional form, we arrive at

$$
\begin{gathered}
\mathrm{Bu}\left[\frac{1}{f T} \frac{\partial \eta}{\partial t}+\operatorname{Ro} \boldsymbol{\nabla} \cdot(\mathbf{u} \eta)\right]+\delta=Q \\
\frac{1}{f T} \frac{\partial \zeta}{\partial t}+\nabla \cdot[\mathbf{u}(\operatorname{Ro} \zeta+1)]=-(\alpha / f) \zeta \\
\frac{1}{f T} \frac{\partial \delta}{\partial t}+\operatorname{Ro} \frac{1}{2} \nabla^{2}(\mathbf{u} \cdot \mathbf{u})+\nabla^{2} \eta-\hat{\mathbf{k}} \cdot \nabla \times[\mathbf{u}(\operatorname{Ro} \zeta+1)] \\
=-(\alpha / f) \delta
\end{gathered}
$$

where we have introduced the Rossby number

$$
\text { Ro } \equiv U /(f L)
$$

the Rossby radius

$$
L_{R} \equiv \frac{\sqrt{g H}}{f},
$$

and the Burger number

$$
\mathrm{Bu} \equiv\left(\frac{L}{L_{R}}\right)^{2} .
$$

From (7), it is easily shown that, if

$$
\max \left(\frac{1}{f T}, \mathrm{Ro}\right) \mathrm{Bu} \ll 1,
$$

then the mass source specifies the divergence at leading order. We have to check, a posteriori, whether our scaling yields a nontrival balance in (8) and (9).

\section{Linear limit}

The linear limit follows by putting Ro $=0$. Then, in the weakly damped, low-frequency limit $(1 /(f T) \ll 1$, $\alpha / f \ll 1)$, no term in the vorticity equation can balance the divergence. Therefore, we have to rescale $\zeta \rightarrow \zeta(f T)$. To get a balance in the divergence equation, we also need to rescale $\eta \rightarrow \eta(f T)$. This imples that the WTG approximation is now valid if $\mathrm{Bu} \ll 1$.

\section{b. Resulting system}

Taking $T \sim \alpha^{-1} \sim f^{-1}$ for the moment, the scaled system is

$$
\begin{aligned}
\delta & =Q, \\
\frac{\partial \zeta}{\partial t}+Q(\operatorname{Ro} \zeta+1)+\mathbf{u} \cdot \nabla(\operatorname{Ro} \zeta+1) & =-\frac{\alpha}{f} \zeta,
\end{aligned}
$$




$$
\begin{aligned}
\nabla^{2} \eta= & -\frac{\partial Q}{\partial t}-\operatorname{Ro} \frac{1}{2} \nabla^{2}(\mathbf{u} \cdot \mathbf{u}) \\
& +\hat{\mathbf{k}} \cdot \nabla \times[\mathbf{u}(\operatorname{Ro} \zeta+1)]-\frac{\alpha}{f} Q .
\end{aligned}
$$

Equation (10), the fundamental WTG approximation, is obviously a diagnostic equation for the divergence if $Q$ is known, a familiar result discussed in the introduction. If $T \gg L / U$, we can drop the tendency terms in (11) and (12). The presence of $Q$ in place of $\delta$ in the vortex stretching term [the second term on the lhs of (11)] shows explicitly that, once the divergence is known from (10), it may be treated as a known forcing. The advecting velocity $\mathbf{u}$ has a divergent part that can be obtained by integration of (10) given boundary conditions; the rotational part must be obtained from the vorticity, for example, by expressing it as the Laplacian of the stream function and inverting the resulting elliptic equation (again requiring boundary conditions). At this point the entire flow is known, and the divergence equation (12) has not been used. It has been written above so as to emphasize that it functions as a diagnostic equation for the small perturbation height $\eta$, all terms on the rhs being known once the preceding two equations have been solved. Note that there is only one true dynamical time derivative in the system, in (11); the one in (12) is a given forcing, since $Q$ is taken given here.

\section{c. Comment on the diabatic scaling}

Balance models have been constructed previously that are valid in the adiabatic case with scaling otherwise similar to ours (Gent and McWilliams 1983a-c; McWilliams 1985; Raymond 1992, 1994; see also Stevens et al. 1990). Generally this leads to a situation in which the divergence is small compared to vorticity, in fact by an even larger margin than in quasigeostrophy (Charney 1963). The key difference here is that our model is inherently diabatic; that is, the heating $Q$ is included a priori in the scaling analysis and taken to be important at leading order. We can see this by noting that the basic scalings [section $2 \mathrm{a}(1)$ ]

$$
\eta \ll H \quad \text { and } \quad H \sim \frac{Q_{0} L}{U}
$$

together imply that

$$
\frac{\eta_{0} U}{Q_{0} L} \ll 1 .
$$

The dimensionless parameter in (13) can be thought of as representing the magnitude of horizontal temperature advection relative to heating. That it is required to be small clearly differentiates the present model from virtually all other balance models, which are derived assuming adiabatic scaling. In the nonlinear case, (13) is equivalent to

$$
\frac{\eta_{0} f}{Q_{0}} \ll 1 .
$$

The model of HH85 differs in a number of particulars from that presented above, most importantly in excluding nonlinearity, in explicitly including the $\beta$ effect, and in using separate meridional and zonal scales. Nonetheless, their model has the same essential dynamical structure as that above, one which is somewhat counterintuitive if one is accustomed to thinking about balanced flows in midlatitudes. In HH85 (and the present work), the heating gives the divergence, which gives the vorticity, which gives the temperature. HH85 commented rather little on the dynamics of their model beyond making that basic point. Some further details warrant discussion in our view. For the most part the same comments would apply to HH85's model.

\section{d. Dynamical properties}

\section{1) BALANCE}

The model derived above is balanced, containing no explicit gravity waves. This is immediately evident from (10), which has no terms containing $\eta$. Further insight is gained into the nature of the balance by inspecting the divergence equation (12). The dynamical time derivative in this equation has been eliminated by using (10); $\partial \delta / \partial t$ has been replaced by $\partial Q / \partial t$, which here is considered given. Note that if $T$ is small compared to $f^{-1}$, consistency cannot be maintained, in that the tendency term in (9) will be larger than all the others. Physically, a heating that varies too rapidly must drive gravity waves. Hence our balanced scaling requires, unsurprisingly, that the heating must not vary on a timescale that is short compared to $f^{-1}$.

\section{2) AdiabAtic LiMit, ENERGy, AND POTENTIAL VORTICITY}

WTG is an inherently nonconservative model, in that the scaling analysis used to derive it assumes that the heating is important at leading order. Because of this, it may be somewhat misleading to consider the properties of the equations in the adiabatic limit $(Q=0)$. However, it is worth doing so in order to place WTG in the context of other balance models.

When $Q=0$, the WTG equations become formally identical to those for strictly 2D incompressible flow; that is, the system obeys pure barotropic vorticity dynamics. As a consequence, if the system is also inviscid $(\alpha=0)$,

1) absolute vorticity is conserved on trajectories,

2) the global integral of the kinetic energy is conserved, and

3) volume is conserved.

In fact, Charney (1963) showed by a scaling analysis that synoptic-scale tropical adiabatic motions should 
obey barotropic vorticity dynamics. The derivation of WTG can be considered an extension of Charney's analysis to the diabatic regime.

When $Q \neq 0$, absolute vorticity is no longer conserved. Neither is potential vorticity $[(\zeta+f) / h]$; in fact, because in both leading order equations [(10) and (11)] $h$ is approximated by $H$, WTG cannot distinguish between absolute and potential vorticity.

Since it does not have a prognostic equation for $h$, a prognostic equation for potential energy cannot be formed under WTG. We can say that potential energy is created by the heating but is immediately converted to kinetic energy. Given these limitations, we can predict confidently (despite the fact that the analysis here is restricted to the shallow water system) that a continuously stratified version of WTG would not support baroclinic instability. This may limit the applicability of WTG to the study of easterly waves in Africa, and perhaps other regions as well (though over the tropical Pacific, e.g., we are not aware of observational evidence for the importance of baroclinic instability).

\section{3) Role OF MOMENTUM DAMPING}

The rotational flow is affected by the Rayleigh friction in the above system, but the divergent flow, being diagnosed directly from continuity, is not. In cases in which the rotational and divergent flows are of the same order (the most prominent exception being the weakly damped, low-frequency, linear limit), this appears to be a formal inconsistency, though not an important one in our view. It results from the fact that the divergent flow has been calculated from what ultimately is a thermodynamic equation rather than a momentum equation. The friction term does appear in the divergence equation as a consequence of this assumption, but this equation has become a diagnostic for $\eta$. Hence the temperature $i s$ affected by friction although the divergent flow is not. This situation is independent of our choice of a Rayleigh friction and would be true for any momentum forcing. The inconsistency presumably will not cause large errors if the damping is small. On the other hand, if $\alpha \gg$ $f$, then we expect that damping, rather than rotation, will allow significant $\eta$ gradients to be sustained, and the scaling will fail even for $\mathrm{Bu} \ll 1$. WTG is fundamentally a nearly inviscid scaling.

\section{4) MAss CONSERVATION}

The truncation of the continuity equation (which plays the role of the thermodynamic energy equation) means that the global conservation properties of the exact equation are not inherently satisfied unless $Q$ happens to integrate to zero over the domain. However, any residual in this integral implies a secular trend in the global mean temperature (represented by $h$ ), so the apparent problem can be remedied by carrying a separate equation for this global mean. In the axisymmetric so- lution below, we simply impose a $Q$ whose global area integral vanishes. In the context of a more physical mod$\mathrm{el}$, in which diabatic processes are explicitly parameterized, appropriate ways of dealing with this issue are discussed by SB00.

\section{5) SCALING UNDER $\beta$}

We have assumed an $f$ plane, but the scaling does not require this. The analysis can be put on an equatorial $\beta$ plane, $f=\beta y$, so that in terms of the scale of variations of $f$ we have $f \sim \beta L$. The scaling in the nonlinear regime $(U / L \sim f)$ then requires $U \sim \beta L^{2}$, so $L$ in this regime is a Rhines scale (Rhines 1975).

\section{e. Axisymmetric solution for top-hat forcing}

Before going on to incorporate moist physics, rendering $Q$ interactive, we present for illustrative purposes the solution to a simple steady-state problem under the dry WTG dynamics with imposed $Q$ of Eqs. (10)-(12). We take $Q$ to be a localized axisymmetric top hat of nondimensional magnitude and radius 1, plus a compensating small negative constant component over a radius $R \gg 1$. As illustrated in Fig. 1a,

$$
Q= \begin{cases}1 & r<1 \\ \gamma & 1<r<R \\ 0 & r>R,\end{cases}
$$

where $\gamma=\left(1-R^{2}\right)^{-1}$ is a small negative number such that the total integral of $Q$ is zero. This is needed to have vanishing flow away from the heating region. It is also realistic if we think of a finite domain with a stationary climate; the integrated heating must vanish if there is to be no secular trend in domain-averaged temperature. We may think of the present forcing profile as representing a localized convective heating plus a spatially uniform radiative cooling. The magnitude and spatial scale of $Q$ are chosen to give Ro $=1$; since the solution is nonlinear, strictly speaking this is a special case.

We solve the problem in polar coordinates, denoting the radial velocity by $u_{r}$ and the azimuthal velocity by $u_{\theta}$. The divergence $\delta$ is immediately obtained from (10), and $u_{r}$ follows from integrating the definition of $\delta$,

$$
\delta=\frac{1}{r} \frac{d\left(r u_{r}\right)}{d r},
$$

yielding

$$
u_{r}(r)= \begin{cases}\frac{1}{2} r & r<1 \\ \frac{1}{2} \gamma r\left[1-(R / r)^{-2}\right] & 1<r<R \\ 0 & r>R .\end{cases}
$$


Notice that $u_{r}$ is continuous everywhere but, since $\delta$ is discontinuous at $r=1, u$ has a kink there (as well as a much smaller kink at $r=R$, where $u_{r}=0$ ).

Having obtained $\delta$ and $u_{r}$, the vorticity equation is now used to solve for the vorticity $\zeta$. Since we are looking for steady-state solutions, the vorticity equation reduces to

$$
\boldsymbol{\nabla} \cdot[\mathbf{u}(\zeta+1)]=-\alpha \zeta,
$$

where $\alpha$ is the nondimensional damping time [written $\alpha / f$ in (8)] and we have taken Ro $=1$. Because all variables are axially symmetric in this problem, the vorticity equation takes the simple form

$$
u_{r} \frac{d \zeta}{d r}+(\delta+\alpha) \zeta=-\delta,
$$

and is easily integrated for $\zeta$, with $\delta$ given by (10) and $u_{r}$ given by (17).

Since $u_{r}$ is proportional to $r$ in the heating region $r$ $<1$, it is readily apparent from (19) that a potential singularity for $\zeta$ could result at the origin. To avoid this, we take $\zeta$ to be a constant in that region and obtain $\zeta(r)$ $=-(1+\alpha)^{-1}\left(\equiv \zeta_{1}\right)$ for $r<1$. Obviously, in the outermost region $\zeta=0$. In the intermediate region $1<r$ $<R$ integration of the first-order ODE (19) yields a single integration constant. Hence $\zeta$ cannot be made continuous at both $r=1$ and $r=R$. We choose to enforce continuity of $\zeta$ at $r=1$. This leaves us with a small discontinuity at $r=R$, whose only damage is to produce a tiny kink in the azimuthal velocity $u_{\theta}$.

The full solution for $\zeta(r)$, plotted in Fig. 1b, is then

$$
\zeta(r)= \begin{cases}\zeta_{1} & r<1 \\ \zeta_{\gamma}+\left(\zeta_{1}-\zeta_{\gamma}\right)\left[\frac{r^{2}-R^{2}}{1-R^{2}}\right]^{\Gamma} & 1<r<R \\ 0 & r>R\end{cases}
$$

with $\Gamma=-(1+\alpha / \gamma)$ and $\zeta_{\gamma} \equiv 1 / \Gamma$. Having obtained $\zeta$ we now integrate

$$
\zeta=\frac{1}{r} \frac{d\left(r u_{\theta}\right)}{d r}
$$

and obtain $u_{\theta}$, the rotational component of the flow,

$$
u_{\theta}(r)= \begin{cases}\frac{1}{2} \zeta_{1} r & r<1 \\ \frac{1}{2} \zeta_{1}\left(1 / r^{2}\right)+\frac{1}{2} \zeta_{\gamma}\left[\left(r^{2}-1\right) / r\right]+\frac{1}{2}\left(\zeta_{1}-\zeta_{\gamma}\right) \frac{1}{\alpha r}\left(1-\left[\frac{r^{2}-R^{2}}{1-R^{2}}\right]^{(\Gamma+1)}\right) & 1<r<R \\ 0 & r>R .\end{cases}
$$

Notice that since $\zeta$ (unlike $\delta$ ) is continuous at $r=1$, the rotational velocity $u_{\theta}$ (unlike the divergent velocity $u_{r}$ ) is smooth at $r=1$. This is clearly seen in Fig. 1c.

To complete the solution, the correction to the height field $\eta$ due to the heating $Q$ may be computed, in general, from the divergence equation. Here, because of axisymmetry, we can obtain it more simply from the radial momentum equation, which in nondimensional form is simply

$$
u_{r} \frac{d u_{r}}{d r}+\frac{u_{\theta}^{2}}{r}-u_{\theta}=-\frac{d \eta}{d r}-\alpha u_{r},
$$

for the axisymmetric steady-state case. Since $u_{r}$ and $u_{\theta}$ are known, we can solve for $\eta$, choosing the integration constant so that $\eta=0$ for $r \geq R$,

$$
\begin{gathered}
\eta(r)=\int_{r}^{R}\left(u_{r} \frac{d u_{r}}{d r}-\frac{u_{\theta}^{2}}{r}-u_{\theta} \alpha u_{r}\right) d r \\
\text { for } r \leq R .
\end{gathered}
$$

This is plotted in Fig. 1d.

The actual analytical expression for $\eta(r)$ is rather cumbersome and thus not particularly illuminating. In the heating region $r<1$, however, it is easily seen that since both $u_{r}$ and $u_{\theta}$ are proportional to $r$, the integrand in (24) is also linear in $r$ and thus $\eta$ is a a simple quadratic, as Fig. 1d attests.

We reiterate that to obtain this WTG solution, terms were dropped only in the continuity (temperature) equation (this is what allows us to get $\delta$ immediately from $Q)$ while all other equations were solved exactly.

It should also be noted that there is a fundamental asymmetry in the problem due to the sign of $f$. We solved the above problem for positive $Q$ with no restriction on the value of $\alpha$. However, if the sign of $Q$ is reversed, there is no physically reasonable steady solution for $\alpha<1$. In this case vortex stretching can increase the vorticity without bound, while for positive $Q$ the same process reduces the absolute vorticity but cannot bring it below zero (taking $f>0$ ) since vortex stretching or compression cannot occur where there is no absolute vorticity. If instead of Rayleigh friction the dissipation is made a stronger function of wind speed, such as a quadratic as in bulk aerodynamic surface drag formulas, then stable steady solutions are possible for negative $Q$ and arbitrarily small damping coefficients, 
a. the heating $Q$

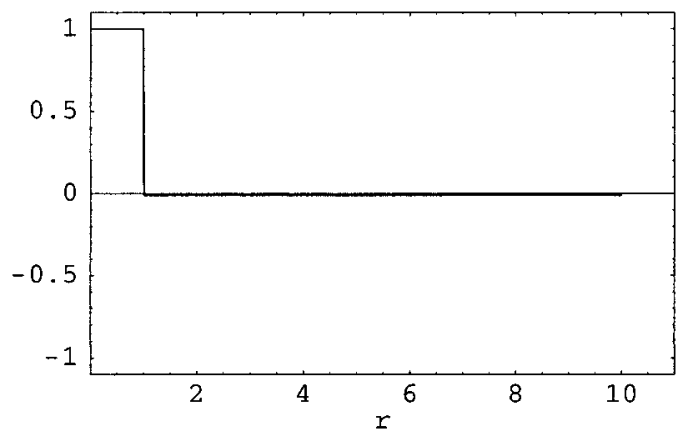

c. $u$ (dashed) and $v$ (solid)

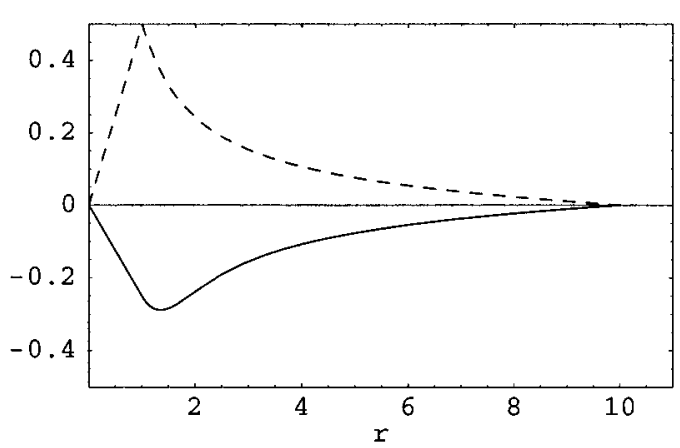

b. $\delta$ (dashed) and $\zeta$ (solid)

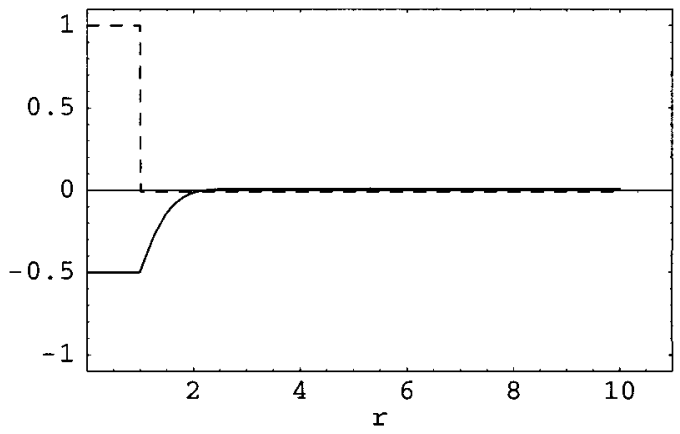

d. the height correction $\eta$

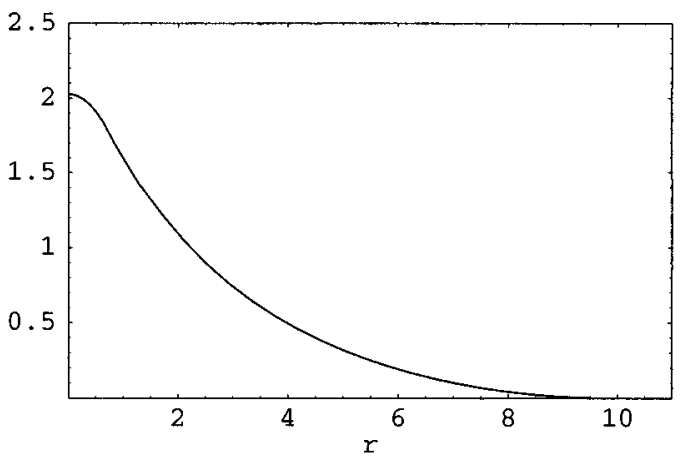

FIG. 1. The flow, computed using the WTG approximation, resulting from a simple axisymmetric heating $Q$ with zero net integral. All variables are identically zero for $r \geq 10$. The case illustrated here is for $\alpha=1$ and $R=10$.

though they may involve very strong circulations. On a $\beta$ plane, weakly damped or even inviscid solutions can be expected to exist for localized forcing of either sign, since energy will be radiated away from the forcing region by Rossby waves.

\section{Balanced waves on an f plane with a background horizontal moisture gradient}

In this section we add a dynamical moisture equation to the shallow water WTG system. The heating is taken proportional to the moisture field, coupling the moisture equation to the others. The system is derived in appendix A from the QTCM equations of NZ00, and our notation retains some features of the QTCM's (resulting in some changes in variable names and new constant coefficients relative to the shallow water system of section 2 ). We also linearize the system to facilitate analysis of its wave propagation mechanisms.

The QTCM framework is useful for this purpose in several ways. The QTCM derivation itself (found in NZ00) illustrates specifically and systematically the degree and kind of physical assumptions that are required in order to obtain a system of this simplicity from the full atmospheric physics and dynamics. The numerical implementation of the QTCM (NZ00; Zeng et al. 2000) demonstrates the climate that these equations, in their full nonlinear form, can produce, providing a valuable link between climate modeling and theory. Finally, the QTCM framework provides a basis for estimating the parameters that appear in the equations below, and some version of which should appear in any analogous system (i.e., a reduction of the primitive equations that includes highly truncated vertical structure and an advected moisture variable).

However, the system below can also be derived by assumptions about the vertical truncation and physical parameterizations different in detail, if not in basic physical interpretation, from those used to derive the QTCM. The proportionality of the heating to the moisture can be viewed in this general context as resulting from linearization about a typical moisture profile of another convective scheme than the simplified Betts-Miller scheme used in the QTCM.

\section{a. Basic state}

Before presenting our moist linear model, we briefly discuss the basic state about which it will be linearized. Our basic states are either horizontally uniform in all fields or contain a constant horizontal gradient in the moisture field only. In order to exhibit the novel wave modes of the system in the clearest way possible, we wish to avoid complications resulting from nontrivial 
basic-state flows; we will allow only a constant barotropic zonal wind in our basic state. Therefore, we assume that the basic state is one of local radiative-convective equilibrium everywhere,

$$
\bar{Q}_{R}+\bar{Q}_{c}=0 \text {, }
$$

with $Q_{R}$ and $Q_{c}$ the radiative and convective heating (in energy units), respectively; and overbars representing basic-state quantities. The moisture budget then requires also that

$$
\bar{Q}_{c}=\bar{E},
$$

with $E$ the evaporation also in energy units. We will assume a basic-state latitudinal moisture gradient, taken constant for simplicity.

\section{b. The moist linear WTG model}

Our linear model, as derived in appendix A, consists of the following equations:

$$
\begin{aligned}
\bar{M}_{S} \delta & =\tau_{c}^{-1} q, \\
\frac{\partial \zeta}{\partial t}+\bar{U}_{0} \frac{\partial \zeta}{\partial x}+f \delta & =-\alpha \zeta, \\
\kappa \nabla^{2} T & =f \zeta-\left(\frac{\partial}{\partial t}+\alpha\right) \delta, \\
\hat{b}\left(\frac{\partial q}{\partial t}+\bar{U}_{0} \frac{\partial q}{\partial x}\right)+v \beta_{q}-\bar{M}_{q} \delta & =-\tau_{c}^{-1} q .
\end{aligned}
$$

All notation is defined in the appendix (which simply restates material from NZ00), but we briefly introduce here a few significant features unfamiliar from section 2 . Dependent variables, without overbars, represent perturbation quantities; these are $\delta, \zeta, T$, and $q$. The flow fields now explicitly represent a baroclinic mode whose sign represents the velocity in the upper troposphere; the flow in the lower troposphere has the opposite sign. Consistent with section $2, \zeta$ and $\delta$ are the vorticity and divergence of this baroclinic flow field. Here $\eta$ is now explicitly relabeled as the temperature $T$; and $\kappa=R /$ $C_{p}$, the ratio of the gas constant for air to the heat capacity of air at constant pressure. The mean depth $H$ becomes the mean dry static stability $M_{S}$. Ignoring these relabelings and new constant coefficients, (25)-(27) are equivalent to linearized versions of (10)-(12).

In the equation for moisture (28), we have defined

$$
\beta_{q}=\langle b V\rangle \frac{\overline{\partial q}}{\partial y},
$$

with the greek letter $\beta$ chosen intentionally to be suggestive. The coefficients $\hat{b}$ and $\langle b V\rangle$ capture the fact that the vertical structure of the flow and moisture fields are not generally identical so that the advection terms require the projection of one upon the other. Both the angle bracket and caret represent a vertical integral; $b(p)$ and $V(p)$ are the vertical structure functions for moisture and baroclinic flow velocity, with $p$ the pressure, and $\langle b V\rangle$ is a negative constant (e.g., $\left\langle b_{1} V_{1}\right\rangle$ in Table 1 of NZ00). Here $M_{q}$ is the gross moisture stratification, which plays a role in the moisture equation analogous to $M_{S}$ in the temperature equation (or to $H$ in the $h$ equation of section 2 ). The "moisture sink" on the rhs of (28) is the negative of the convective heat source on the rhs of (25). This reflects the simplest moisture closure for the QTCM, of several discussed by NZ00.

Here $\tau_{c}$ is a convective timescale, typically taken on the order of hours. It can be argued that the moisture field cannot react to convection nearly as quickly as the buoyancy field can and, thus, that the use of identical timescales in the moisture and temperature equations is inappropriate. For simplicity we have left this feature of the Betts-Miller scheme as is. However, the linear modes we obtain below are sensitive to $\tau_{c}$, and so the possibility that a timescale on the order of days, rather than hours, could enter should be kept in mind.

To further isolate the role of horizontal moisture advection, we have assumed that the perturbations have a negligible effect on the radiative cooling and surface latent heat flux,

$$
Q_{R}^{\prime}=E^{\prime}=0 .
$$

We have also neglected horizontal diffusion terms in the advection-diffusion operators and allowed a constant barotropic wind in the basic state, $\bar{U}_{0}$.

Apart from the moisture equation (28), the above system (25)-(27) is isomorphic to the linear shallow water WTG system forced by a mass source given by a linearization of (10)-(12), with the mass source now controlled by the moisture. With the addition of (28), our linear model contains two true dynamical equations, for moisture and baroclinic vorticity. Correspondingly, in general we expect the dispersion relation to have two sets of solutions, or "modes."

\section{c. Results}

\section{1) IRRotATIONAL MODES}

The vorticity is coupled to the divergence through the action of vortex stretching on the planetary vorticity $f$. In the case $f=0$ there can be a divergent flow with no associated rotational flow. Modes in this regime can be studied using the thermodynamic equations alone. Thus in this special case we retain only a single time derivative and will obtain only a single mode. Using (25) in (28) we obtain

$$
\hat{b}\left(\frac{\partial q}{\partial t}+\bar{U}_{0} \frac{\partial q}{\partial x}\right)+v \beta_{q}=-\frac{\bar{M}}{\tau_{c} \bar{M}_{S}} q,
$$

in which $\bar{M}=\bar{M}_{S}-\bar{M}_{q}$ is the basic-state gross moist stability, which can be assumed to be small compared to $\bar{M}_{S}$ and $\bar{M}_{q}$ separately, but positive (Neelin and Held 
1987; Yu and Neelin 1998). Notice that since both (25) and (28) were used in obtaining (29), the latter is really a perturbation moist static energy equation, but one in which temperature perturbations are neglected in favor of moisture perturbations.

(i) The case $\beta_{q}=0$

One interesting case that immediately reveals itself is that of no basic moisture gradient, $\beta_{q}=0$. In that case (29) becomes

$$
\frac{\partial q}{\partial t}+\bar{U}_{0} \frac{\partial q}{\partial x}=-B q
$$

where we have defined

$$
B \equiv \frac{\bar{M}}{\hat{b} \tau_{c} \bar{M}_{S}},
$$

and in which the Lagrangian tendency is proportional to the mean gross moist stability, in the sense of a damping as long as $M$ is positive. Physically, $M$ in (30) controls the difference between the precipitation and the moisture convergence, and hence the Lagrangian humidity tendency. This is a rather different role for $M$ than the one that it plays in quasi-equilibrium theories of convection interacting with gravity waves, where $M$ is proportional to the square of the wave frequency (e.g., Emanuel et al. 1994).

In the limit as $M \rightarrow 0$ we obtain the simple conservation relation,

$$
\frac{\partial q}{\partial t}+\bar{U}_{0} \frac{\partial q}{\partial x}=0
$$

Upon first glance this relation may seem trivial, but it is not, since it does not represent conservation in the most standard sense; rather, it represents a cancellation of opposite nonconservative effects. The moisture perturbations are associated with perturbations in convective heating and hence precipitation, but the loss of moisture in precipitation is balanced by moisture convergence.

Here $B$ has units of inverse time. Generally we expect $M / M_{S}$ to be less than unity but not by more than an order of magnitude, while $\hat{b}=0.45$ according to (the value for $\hat{b}_{1}$ in) Table 1 of NZ00. ${ }^{2}$ Therefore, the timescale $B^{-1}$ can be considered in convective regions to be of the same order as the convective timescale, hours to days (if the potentially longer moisture timescale is considered). The $M=0$ regime may nonetheless be qualitatively reasonable in a local sense as a representation of very moist regimes in which $M \ll M_{S}$.

\footnotetext{
${ }^{2}$ However, this value depends on the particular basis functions chosen for moisture and baroclinic velocity, and so cannot be regarded as precisely known.
}

(ii) The case $\beta_{q} \neq 0$

Since (25) relates the divergence and moisture perturbations in a simple way, (28) can be turned into an equation for the divergence, and it proves advantageous to do this at this point. We define a velocity potential by the standard relation

$$
\nabla^{2} \chi=\delta
$$

Using these relations to convert (28) into a $\chi$ equation, we obtain

$$
\left(\frac{\partial}{\partial t}+\bar{U}_{0} \frac{\partial}{\partial x}+B\right) \nabla^{2} \chi+A \frac{\partial \chi}{\partial y}=0,
$$

where we have defined

$$
A=\frac{\beta_{q}}{\hat{b} \tau_{c} \bar{M}_{S}} .
$$

Here $A$ has units of inverse time over length so that we can define a length scale as

$$
L_{q}=B / A=M / \beta_{q} .
$$

Here $L_{q}$ can be physically interpreted as the length scale over which $M$ varies at $O(1)$ due to the basic-state moisture gradient. Since our linear analysis takes $M$ constant, this indicates that the linearization is only formally justifiable for motions whose meridional scales are small compared to $L_{q}$. With parameters taken from NZ00 and typical values of $M$ and $q$ taken from a climatological simulation with their model, taking $\beta_{q}$ to represent the average moisture gradient between the equator and subtropics, a value for $L_{q}$ on the order of $3000 \mathrm{~km}$ is obtained.

We assume solutions to (32) of the form

$$
\chi \propto e^{i(\omega t-l y)}
$$

and to take $U_{0}=0$. In this case the dispersion relation obtained, a special case of (39) below, has a root given by

$$
\omega=A\left(i L_{q}-\frac{1}{l}\right) .
$$

This mode has phase propagation up the moisture gradient and is damped on the timescale $B^{-1}$.

\section{2) Rotational modes}

We now turn to the case $f \neq 0$, in which the divergent flow induces a rotational flow. We express the rotational flow in terms of a streamfunction $\psi$ so that, in general,

$$
\mathbf{v}=\boldsymbol{\nabla} \chi+\nabla \times \hat{\mathbf{k}} \psi,
$$

where

$$
\nabla^{2} \psi=\zeta
$$

With this, our moisture equation becomes 
$\operatorname{REAL}(\omega)$

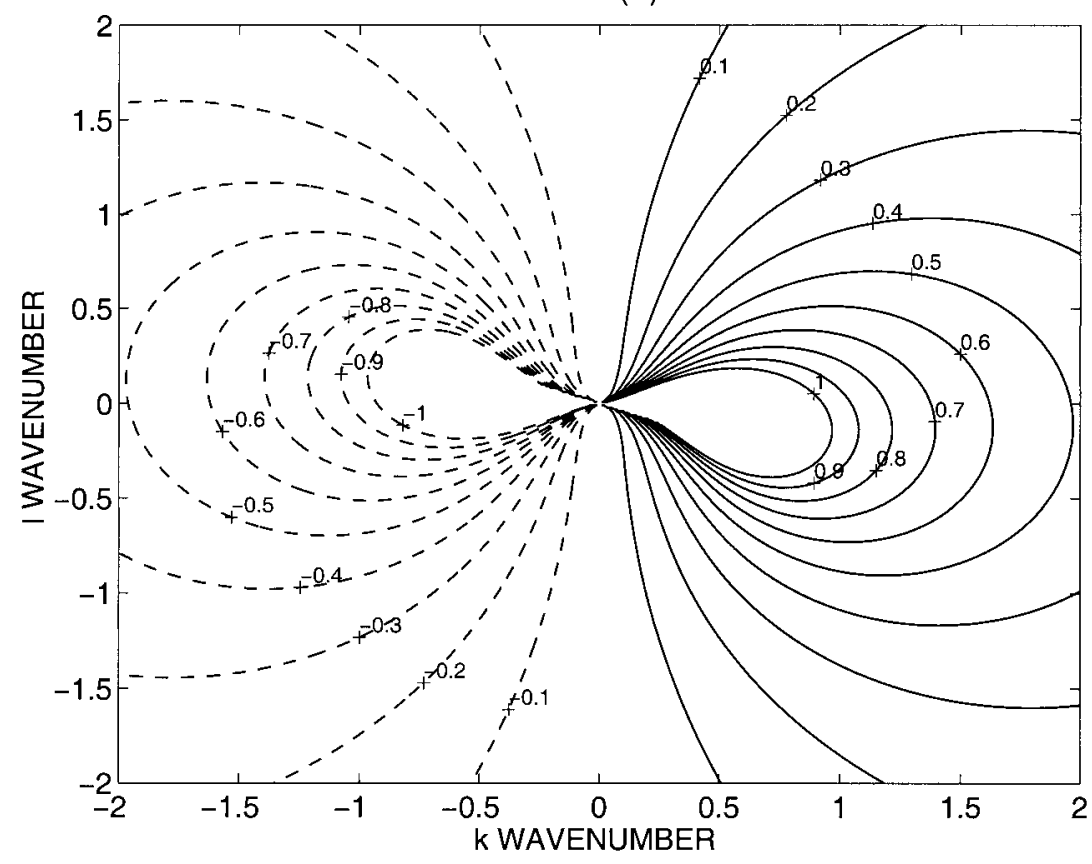

FIG. 2. Real part of the frequency for the rotational modes, as a function of meridional and zonal wavenumbers.

$$
\left(\frac{\partial}{\partial t}+\bar{U}_{0} \frac{\partial}{\partial x}+B\right) \nabla^{2} \chi+A\left(\frac{\partial \psi}{\partial x}+\frac{\partial \chi}{\partial y}\right)=0
$$

while writing the vorticity equation (26) in terms of a streamfunction we have

$$
\left(\frac{\partial}{\partial t}+\bar{U}_{0} \frac{\partial}{\partial x}\right) \nabla^{2} \psi+f \nabla^{2} \chi=-\alpha \nabla^{2} \psi
$$

From this point on, in order to most clearly illuminate the novel mechanisms operating in this system, we make the simplifying assumptions $U_{0}=\alpha=0$. Equation (35) becomes simply

$$
\nabla^{2}\left(\frac{\partial \psi}{\partial t}+f \chi\right)=0
$$

which, except in trivial cases, implies that

$$
\frac{\partial \psi}{\partial t}=-f \chi
$$

For frequencies small compared to $f$, (37) implies $\zeta \gg$ $\delta$, as discussed at the end of section $2 \mathrm{a}(1)$. Using (37) to eliminate $\psi$ from (34), we obtain

$$
\frac{\partial}{\partial t}\left(A^{-1} \frac{\partial}{\partial t}+L_{q}\right) \nabla^{2} \chi=-\frac{\partial^{2} \chi}{\partial y \partial t}+f \frac{\partial \chi}{\partial x}
$$

and assuming wavelike solutions with frequency $\omega$ and wavenumber vector $(k, l)$,

$$
\chi \propto e^{i(\omega t-k x-l y)},
$$

this results in the dispersion relation

$$
A^{-1} \omega^{2}-\omega\left(i L_{q}-\frac{l}{K^{2}}\right)+\frac{i f k}{K^{2}}=0,
$$

where $K^{2}=k^{2}+l^{2}$.

One branch of (39) is a rotational counterpart of that discussed in the preceding section; one easily sees that for sufficiently large $\omega$ and $k=0$, (39) becomes (33). This mode is damped on the timescale $B^{-1}$. For reasonable parameters, this mode has frequencies high enough that the basic WTG scaling becomes questionable [section $2 \mathrm{~d}(1)$, and below], although this depends on $\beta_{q}$ among other parameters and so is not a general conclusion. For these reasons, this fast mode may not be physically significant, but this is not entirely clear, as it depends (among other things) on the effective $B^{-1}$ that would obtain in a model with a model with a separate, large timescale for moisture. If desired, this mode can be eliminated from the equations at the outset by dropping the tendency term in (28). This renders the moisture a diagnostic variable, proportional to the meridional velocity $v$.

The other branch corresponds to a slow mode, for which the real and imaginary parts of $\omega$ are contoured in Figs. 2 and 3 as functions of $k$ and $l$, with length nondimensionalized by $L_{q}$ and time by $f^{-1}$ so that (39) becomes

$$
\left(\frac{f}{B}\right) \omega^{2}-\omega\left(i-\frac{l}{K^{2}}\right)+\frac{i k}{K^{2}}=0,
$$




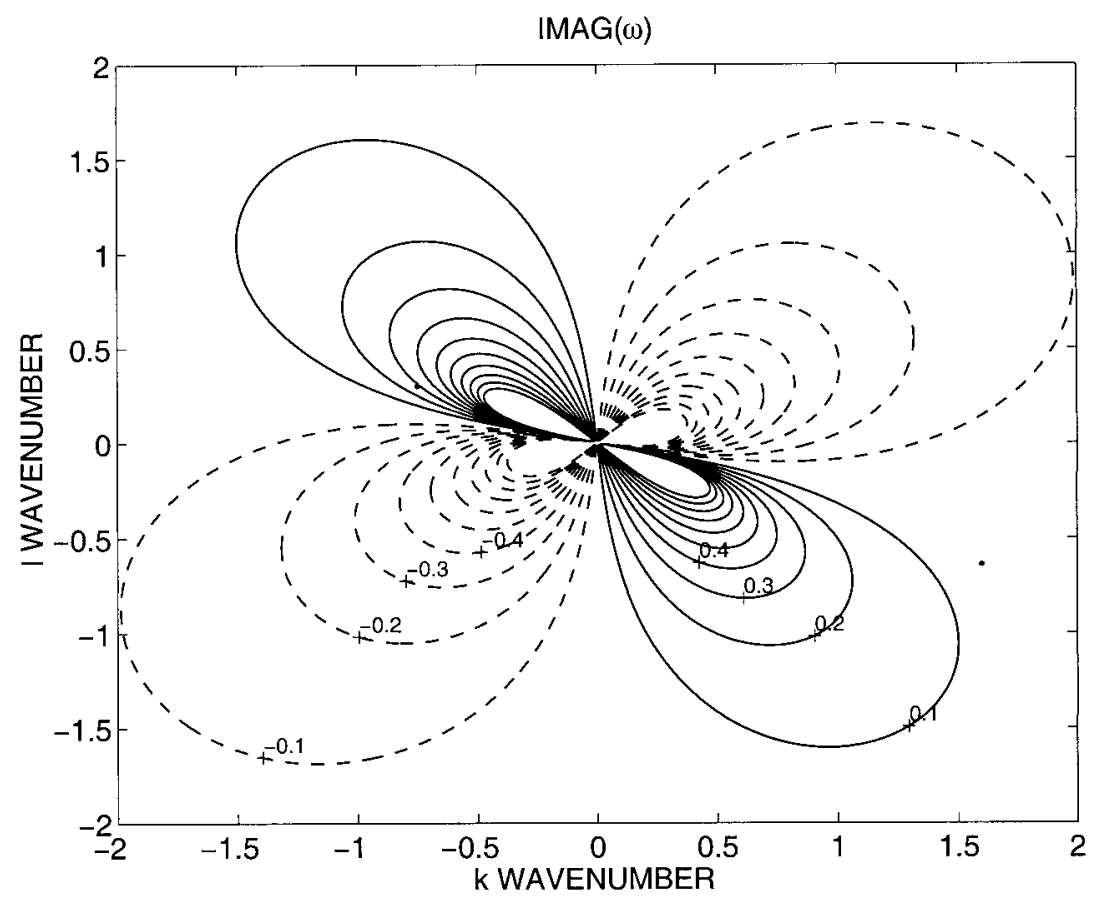

FIG. 3. Imaginary part of the frequency for the rotational modes, as a function of meridional and zonal wavenumbers.

in terms of the nondimensional $\omega, k$, and $l$. We see that there is one nondimensional parameter that must be specified, expressed in (40) as $f / B$. Based on a plausible (but far from precise) tropical scaling, we give this parameter the value 0.2 for plotting purposes.

To see the character of the slow mode, note that we can neglect the quadratic term in (40) for sufficiently small $f / B$. In dimensional terms, we can neglect the quadratic term relative to $i L_{q} \omega$ in (39) when $\omega \ll B$, which by the estimate above corresponds to periods longer than a day or so. Alternatively, this term is eliminated by dropping the tendency term in (28), which also eliminates the fast mode as noted above. In this limit the dispersion relation becomes (for the moment, assuming that the product $A l$ is also large relative to the dimensional $\omega^{2}$ )

$$
\omega \approx \frac{f k}{L_{q} K^{2}+i l} .
$$

In this approximation, the broken symmetry (or antisymmetry) of the frequency relative to the $k$ and $l$ axes, shown in Figs. 2 and 3 is restored.

A special case of interest is $l=0$, in which (41) reduces to

$$
\omega \approx \frac{f}{L_{q} k}=\frac{\beta_{q} f}{\bar{M} k},
$$

which bears an obvious similarity to the dispersion relationship for a Rossby wave with $l=0$ on a standard $\beta$ plane, though the wave propagation mechanism is quite different.
One interesting feature of (42) is its sign. If $\beta_{q}$ is positive, corresponding to decreasing humidity toward the Pole, the intrinsic phase velocity of the waves is eastward, the opposite of the standard Rossby wave. We may understand the propagation mechanism by considering the phase relations for a low-frequency wave that has the background moisture gradient drier toward the pole. A positive moisture anomaly in such a wave will be collocated with a positive low-level $v$ anomaly. This would seem to amplify the wave, but it does not since the moisture advection is balanced by "damping" at the rate $B$. The low-level vorticity maximum is approximately one quarter-cycle west of the moisture maximum, but the low-level convergence maximum, which is collocated with the moisture maximum, creates a maximum in the vorticity tendency there, causing eastward propagation.

A second interesting feature of (42) is the inverse dependence of the frequency on the background gross moist stability, in contrast to the role this parameter plays in theories of gravity waves coupled to convection (e.g., Emanuel et al. 1994). A third is that the convective timescale $\tau_{c}$ does not appear in (42).

Turning to the growth rates, as plotted in Fig. 3, we see that within a regime near $k \approx l$, for $k$ and $l$ both of order unity or smaller (nondimensionalized by $L_{q}$ ), the growth rate (nondimensionalized by $f$ ) of this slow mode is significant. In fact, the growth rate has an "infrared catastrophe." This is most quickly understood by considering the limit $f / B \rightarrow 0$ (but fixed $L_{q}$ ) as discussed above. In this limit, the nondimensional growth rate is 


$$
-\operatorname{Im}(\omega)=\frac{k l}{K^{4}+l^{2}},
$$

which blows up at the origin. However, the growth rates only become extremely large for wavenumbers much larger than the circumference of the earth, a geophysically irrelevant regime. Also, as mentioned above, the linearization of the moisture equation is only strictly valid for $l \gg 1$. Both viscous damping and the $\beta$ effect, if included in the analysis, would reduce the growth rate substantially, though neither would eliminate the singularity at the origin.

\section{3) BRIEF COMMENT ON THE ADDITION OF THE $\beta$ EFFECT}

Given that the standard $\beta$ effect would lead to westward propagation where our " $\beta_{q}$ effect" leads to eastward propagation, it is desirable to quantify the relative importance of these two effects. A fuller analysis of the $\beta$-plane case is under way and will be presented in the near future, including a thorough study of the stability properties and using, as is most appropriate, the equatorial $\beta$ plane. For the moment we only briefly address the issue of most immediate relevance here, which is the direction of phase propagation for both $\beta$ and $\beta_{q}$ nonzero.

For this purpose we use a midlatitude $\beta$ plane, $f=$ $f_{0}+\beta y$, where $y$ is assumed small enough that $\beta$ is only kept in the meridional vorticity advection term, so that no nonconstant coefficients are introduced. In this case (36) becomes

$$
\nabla^{2}\left(\frac{\partial \psi}{\partial t}+f \chi\right)+\left(\frac{\partial \psi}{\partial x}+\frac{\partial \chi}{\partial y}\right) \beta=0 .
$$

Following through on the calculation above with this modification, and taking the low-frequency limit of the resulting dispersion relation, results in

$$
\omega=\frac{k\left(f_{0}-L_{q} \beta\right)}{L_{q} K^{2}+i\left(1+A^{-1} \beta k\right)} .
$$

This shows that the ratio of the restoring stiffness of the $\beta q$ and $\beta$ terms is

$$
\frac{f_{0}}{\beta L_{q}}=\frac{R_{e} \tan \phi}{L_{q}},
$$

which for $L_{q} \sim 3000 \mathrm{~km}$, and $\phi$ on the order of $10^{\circ}$, is of order unity. Given the uncertainties in this estimate it is actually somewhat difficult to assess which effect will be the more important and, hence, in which direction the resulting disturbances will propagate.

\section{Discussion}

\section{a. Comparison with other balance models}

Both in dry and moist implementations, the WTG dynamics is balanced in a sense similar to that of other balance models, although the balance occurs fundamentally in the temperature rather than momentum equation. The model has no explicit gravity waves for the same reason that, for example, quasigeostrophy does not; they are assumed to be infinitely fast. The differences are that in WTG dynamics the adjustment process is assumed to occur in an atmosphere that is rotating weakly at most, and is assumed to be a response to a sustained heating rather than an initial imbalance, which may occur in either the mass or momentum fields. The adjustment process in our case might be considered "convective adjustment," as described by Bretherton and Smolarkiewicz (1989), rather than geostrophic adjustment, but the essence of the process is the same.

In the moist model, we find further analogies to traditional balance models. Most interesting is the fact that the moisture field plays a role more than a little reminiscent of potential vorticity. Under quasigeostrophy, the vorticity can be expressed as the Laplacian of the streamfunction, so that on an $f$ plane, if $P$ is the perturbation quasigeostrophic potential vorticity, we have

$$
\nabla^{2} \psi \sim P
$$

while the assumptions of section $3 \mathrm{~b}$ lead to

$$
\nabla^{2} \chi \sim q
$$

with $\chi$ now the velocity potential and $q$ the perturbation moisture. From this and (35) we see that in the limit of low frequency and large friction, we obtain an even closer correspondence,

$$
\nabla^{2} \psi \sim q
$$

Perhaps the more interesting limit, though one less directly analogous to quasigeostrophic theory, is that discussed in section $3 c(2)$ : small friction, low frequency, and large $B$, in which

$$
v=\frac{\partial \chi}{\partial x}+\frac{\partial \psi}{\partial y} \sim q .
$$

In quasigeostrophic theory $P$ is conserved following adiabatic inviscid geostrophic flow. In our analysis $q$ is not generally conserved, except in the special case of no evaporation anomalies and the limit of vanishing gross moist stability; these are probably not generally good assumptions, but the existence of this limit at least points to the fact that horizontal advection (the dominant process in quasigeostrophic dynamics) plays a role in determining the $q$ field.

\section{b. Qualifications}

The relevance of the dynamical modes derived here to the real atmosphere bears discussion. One may be tempted, as is always the case with models using any particular convective closure scheme, to question how strongly the results depend on the characteristics of that scheme. We cannot answer that question with precision, but at a qualitative level the main feature that is nec- 
essary for modes resembling those described here to exist is that convective heating, all else being equal, be an increasing function of the moisture content of the atmosphere. This is, presumably, a rather generic feature of all reasonable convective schemes, though its quantitative importance may vary from one scheme to the next.

Another limitation inherent in the moist model used here is the single moisture variable with a fixed vertical structure. It is natural, and is the case in the QTCM, that the vertical structure function for moisture projects fairly strongly on boundary layer moisture, which is directly related to convective available potential energy (CAPE) in virtually any formulation. The vertical structure function also projects on free tropospheric relative humidity. The latter is generally presumed to affect convection through entrainment of environmental air into clouds, with buoyancy being more strongly decreased the drier the environment. However, there is also evidence that air above the boundary layer can participate directly in convective circulations, particularly in active mesoscale convective systems (Kingsmill and Houze 1999a,b; see also Zipser et al. 1981; Jorgensen et al. 1991), suggesting that environmental humidity can affect the convection through mesoscale dynamics somewhat differently than the standard picture of entrainment into an isolated cloud. As the effect of environmental humidity at different levels on convection is not well quantified at present, the limitation to a single vertical degree of freedom, with attendant vagueness about which level is most important and how, seems appropriate. However, it does bear stating that free tropospheric humidity can generally be expected to be more strongly affected by horizontal advection than boundary layer humidity, since the latter is more directly controlled by relatively fast processes (Betts and Ridgway 1989).

\section{c. Accuracy}

While the scaling analysis used to derive the WTG system can guide our expectations regarding the accuracy of WTG solutions compared to those of more fundamental equations, we have not addressed this here by direct comparison with more exact solutions. Work aimed at addressing this question is currently well underway in the context of classic models of tropical atmospheric dynamics, such as the Gill model (1980) and simple axisymmetric models of the Hadley circulation. The results so far are encouraging, and this work will be reported in due course. Further discussion is outside our present scope.

\section{d. Possible observational relevance}

The eastward-propagating moist mode derived in section $3 c(2)$ was obtained as the result of a theoretical investigation, rather than being a conscious attempt to explain any particular observed phenomenon. Nonetheless, we present a couple of tentative speculations here on its possible relevance to the real atmosphere. Careful data analyses will be required to determine whether these speculations have merit.

One is tempted by the eastward propagation to suggest that our rotational moisture waves may have some relevance to the Madden-Julian oscillation (MJO), although the addition of the $\beta$ effect complicates this matter, as discussed in section $3 \mathrm{c}(3)$. This application also seems inconsistent with the common interpretation of the MJO as having a fundamentally gravity or Kelvin wave-like aspect. Our mechanism may, however, be consistent with the recent work of Raymond (2001), who suggests, based on his numerical simulations, that the MJO may be more fundamentally rotational, and less Kelvin wave-like, than previous work has suggested. Raymond sees evidence in his simulations for a wave propagation mechanism very similar to that of the slow mode in section $3 \mathrm{c}(2)$.

Perhaps more likely than relevant to the MJO proper, our mechanism may be relevant to aspects of intraseasonal variability in Northern Hemisphere summer, when the centers of convective activity are displaced well off the equator and the associated flows are consequently more rotational in character. We are thinking here of the "convection seesaw" discussed by Zhu and Wang (1993), and to the related monsoon onset and break periods over the Asian continent and tropical western North Pacific discussed by Murakami and Matsumoto (1994). The relevance of both moisture advection and vorticity dynamics to the Asian monsoon onset is clearly suggested by the simulations of Xie and Saiki (1999).

Another possible application is to the propagation of African easterly wave disturbances across the Atlantic basin (e.g., Reed et al. 1988a,b). Given the rotational nature of these disturbances, it has not been obvious how they stay localized against Rossby wave dispersion. One possibility is that our moisture wave mechanism is operating on the moisture gradient between the air masses advected from the Sahara and the moister landmass to its south. This would reduce the "effective $\beta$ " and hence inhibit dispersion. In this context it is worth pointing out that the horizontal advection of the background moisture gradient acts in our slow mode as a distinct effect from the WTG dynamics per se, and one that will also exist in a fully baroclinic system. One might, for that matter, also expect this effect in moist midlatitude disturbances in the vicinity of a strong horizontal moisture gradient, although WTG will not be appropriate for analyzing this situation.

\section{Conclusions}

We first derived the basic WTG scaling for dry shallow water dynamics subject to an imposed mass source, representing a heating. This results in a balanced system fundamentally similar to that derived by $\mathrm{HH} 85$. The 
fundamental balance assumption occurs in the continuity (temperature) equation and, in fact, no additional approximations are needed. Physically, this assumption is that horizontal temperature gradients are negligible despite spatial variations in diabatic heating. In the resulting system, the vorticity equation retains the only dynamical time derivative, and the divergence equation becomes a diagnostic equation for the height (temperature). The scaling requires that the Burger number be small, but the dynamics is fundamentally different from that obtained in this regime in the adiabatic case. The key feature in the WTG system is the a priori importance of diabatic heating (mass source $Q$ ), which can be expressed by defining a small nondimensional parameter containing a $Q$ scale in the denominator.

We then applied this scaling to a linear moist model on an $f$ plane. The key new feature that is added to the shallow water dynamics here is an independent equation for conservation of water. The moisture in turn controls the convective heating and through this governs the balanced dynamics in a manner somewhat reminiscent of (though, in important ways, different than) potential vorticity in classic balance models such as quasigeostrophy. Linear analysis on simple, artificially constructed basic states brings out the existence of modes of variability due fundamentally to horizontal moisture advection to which, as far as we know, no analogs have been previously studied theoretically.

In the low-frequency limit with a background moisture gradient, a mode exists that is identical to the barotropic Rossby wave in mathematical form, though it relies on a different physical mechanism and has phase propagation toward the east. This mode has a significant growth rate over a finite range of (low) wavenumbers. We tentatively suggest that this mode may have some relevance to intraseasonal variability and easterly waves. An interesting problem that may complicate attempts to evaluate the validity of these suggestions is that the mode's phase speed in the presence of the $\beta$ effect is ambiguous in both sign and magnitude for reasonable parameter choices, due to cancellation between the restoring effects of $\beta$ and the moisture gradient.

Acknowledgments. An e-mail discussion with Dr. K. A. Emanuel helped to inspire the analysis in section 3. Drs. C. S. Bretherton (who suggested the name "weak temperature gradient approximation”), A. J. Majda, D. J. Raymond, and J.-I. Yano commented insightfully on the manuscript in advance of submission, leading to a number of improvements. We also thank M. A. Cane, N. Harnik, I. M. Held, D. J. Muraki, J. D. Neelin, R. A. Plumb, and B. Stevens for discussions. AHS acknowledges support from NSF Grant ATM-0096195, and from a David and Lucile Packard Foundation Fellowship for Science and Engineering. A critical portion of the work was done during a visit by AHS to Stockholm University in August 2000, sponsored by the In- ternational Meteorological Institute in Stockholm. LMP acknowledges support from NSF.

\section{APPENDIX}

\section{QTCM Equations as Basis for the Moist Model}

The QTCM equations are derived and discussed at some length in Neelin (1997) and NZ00. We give only a minimal introduction to them here and refer the reader to these studies and the references therein for further information. Numerical solutions to the equations (or approximate versions of them) under various conditions are presented in Zeng et al. (2000), Lin et al. (2000), Chou et al. (2000), and SB00.

The full QTCM equations contain two vertical modes, one barotropic and one baroclinic. A flat bottom surface and flat rigid lid are assumed so that the barotropic mode, denoted by the subscript 0 , is nondivergent. The baroclinic mode is denoted by the subscript 1 and assumes a specified vertical structure, consistent with a quasi-equilibrium convective parameterization based on the Betts-Miller scheme (Betts 1986; Betts and Miller 1986). Hence, the threedimensional fields of velocity $\mathbf{v}$, temperature $T$, and moisture $q$ are described by

$$
\begin{aligned}
\mathbf{v}(x, y, p, t) & =\mathbf{v}_{0}(x, y, t)+V_{1}(p) \mathbf{v}_{1}(x, y, t) \\
T & =T_{r}(p)+a_{1}(p) T_{1}(x, y, t) \\
q & =q_{r}(p)+b_{1}(p) q_{1}(x, y, t),
\end{aligned}
$$

where $T_{r}$ and $q_{r}$ are reference profiles; the vertical structure functions $a_{1}, b_{1}$, and $V_{1}$ are shown in Fig. 1 of Zeng et al. (2000). With this truncation the dynamics is reduced to a set of two-dimensional equations for the scalars $T_{1}$ and $q_{1}$ and velocities $\mathbf{v}_{0}$ and $\mathbf{v}_{1}$.

The barotropic mode satisfies a vorticity equation,

$$
\frac{\partial \zeta_{0}}{\partial t}+\boldsymbol{\nabla} \times \mathcal{D}_{v 0}\left(\mathbf{v}_{0}, \mathbf{v}_{1}\right)=-\boldsymbol{\nabla} \times\left(\epsilon_{0} \mathbf{v}_{0}+\epsilon_{10} \mathbf{v}_{1}\right),
$$

where $\zeta_{0}$ is the barotropic vorticity, $\nabla \times$ is the twodimensional curl, and other notation is as in NZ00. An $f$ plane has been assumed already so that there is no $\beta$ term. The barotropic mode has no direct interaction with the thermodynamics, so there is no temperature or moisture field (i.e., $q_{0}$ or $T_{0}$ ) associated with this mode. If we linearize the QTCM equations and set $\epsilon_{10}=0$, we can easily show that, if there is no barotropic flow at some initial time, there will never be any due to baroclinic forcing. Therefore, we neglect the barotropic mode in our linear model, except that we will allow for a spatially and temporally uniform basic-state barotropic wind.

For the baroclinic mode the equations are, for temperature, 


$$
\begin{gathered}
\hat{a}_{1}\left(\frac{\partial T_{1}}{\partial t}+\mathcal{D}_{T 1} T_{1}\right)+M_{s 1} \mathbf{\nabla} \cdot \mathbf{v}_{1} \\
\quad=\epsilon_{c}\left(q_{1}-T_{1}\right)+Q_{R}+H
\end{gathered}
$$

for moisture,

$$
\begin{aligned}
& \hat{b}_{1}\left(\frac{\partial q_{1}}{\partial t}+\mathcal{D}_{q 1} q_{1}\right)-M_{q 1} \boldsymbol{\nabla} \cdot \mathbf{v}_{1} \\
& \quad=-\epsilon_{c}\left(q_{1}-T_{1}\right)+E
\end{aligned}
$$

and for baroclinic momentum,

$$
\begin{aligned}
\frac{\partial \mathbf{v}_{1}}{\partial t} & +\mathcal{D}_{v 1}\left(\mathbf{v}_{0}, \mathbf{v}_{1}\right)+f \mathbf{k} \times \mathbf{v}_{1} \\
= & -\kappa \nabla T_{1}-\epsilon_{1} \mathbf{v}_{1}-\epsilon_{01} \mathbf{v}_{0} .
\end{aligned}
$$

Notation for the most part is as in NZ00. Here $T_{1}$ and $q_{1}$ are in energy units and represent deviations from fixed reference profiles. Baroclinic wind is defined by $\mathbf{v}_{1}=\left(u_{1}, v_{1}\right)$. The vertical structure function $V_{1}(p)$ is defined so that the sign of $\mathbf{v}_{1}$ is that of the upper-tropospheric wind; the lower-tropospheric wind, which is predominantly responsible for advecting moisture, has the opposite sign. A caret represents a vertical integral, so that $\hat{a}_{1}$ and $\hat{b}_{1}$ are constants (given in Table 1 of NZ00). Here (unlike in section 2) $\kappa=R / C_{p}$, where $R$ is the gas constant for air and $C_{p}$ is the heat capacity of air at constant pressure. The operators $\mathcal{D}_{v 0}, \mathcal{D}_{T 1}, \mathcal{D}_{q 1}$, and $\mathcal{D}_{v 1}$ are fully nonlinear advection-diffusion operators projected on the appropriate basis functions; the detailed form of these is given in Eqs. (5.11)-(5.14) of NZ00. Here $\epsilon_{0}, \epsilon_{10}, \epsilon_{1}$, and $\epsilon_{01}$ are vertical momentum transfer coefficients, which may depend on space and time, and whose form is given in Eq. (5.15) of NZ00. The variables $E$ and $H$ represent surface latent and sensible heat fluxes [Eqs. (5.16)-(5.17) of NZ00], and $Q_{R}$ is the net radiative heating [section $4 \mathrm{f}$ of NZ00; see also Zeng et al. (2000) and Chou and Neelin (1996)]. Here $M_{s 1}$ and $M_{q 1}$ are the dry static stability and gross moisture stratification, which are functions of $T_{1}$ and $q_{1}$ respectively. The variable $\epsilon_{c}$ is defined by

$$
\epsilon_{c}(x)=\tau_{c}^{-1} \mathcal{H}(x)
$$

where $\tau_{c}$ is a convective timescale and $\mathcal{H}(x)$ is a Heaviside function, so that the convective heating (which is represented by the term containing $\epsilon_{c}$ ) is positive only, and otherwise proportional to CAPE projected on the basis functions, $q_{1}-T_{1}$ under the simplest assumptions (see NZ00 for further discussion). Note that $\epsilon_{c}$ is not a small parameter, nor a viscous damping coefficient; the notation follows NZ00.

If we linearize the QTCM equations, make the WTG approximation, set $f=$ constant, set all momentum transfer coefficients except $\epsilon_{1}$ (which is relabeled $\alpha$ in section $3 \mathrm{~b}$ ) to zero, ignore the barotropic mode, and drop all subscripts " 1 " on variables and constants in the baroclinic mode, we arrive at the linear model in section $3 b$.

\section{REFERENCES}

Betts, A. K., 1986: A new convective adjustment scheme. Part I: Observational and theoretical basis. Quart. J. Roy. Meteor. Soc., 112, 677-691.

__ and M. J. Miller, 1986: A new convective adjustment scheme. Part II: Single column tests using Gate wave, BOMEX, ATEX and arctic air-mass data sets. Quart. J. Roy. Meteor. Soc., 112, 693-709.

—_ and W. Ridgway, 1989: Climatic equilibrium of the atmospheric convective boundary layer over a tropical ocean. J. Atmos. Sci., 46, 2621-2641.

Bretherton, C. S., and P. K. Smolarkiewicz, 1989: Gravity waves, compensating subsidence, and entrainment and detrainment around cumulus clouds. J. Atmos. Sci., 46, 740-759.

Charney, J. G., 1963: A note on large-scale motions in the tropics. J. Atmos. Sci., 20, 607-609.

Chen, S. S., R. A. Houze Jr., and B. E. Mapes, 1996: Multiscale variability of deep convection in relation to large-scale circulation in TOGA COARE. J. Atmos. Sci., 53, 1380-1409.

Chou, C., and J. D. Neelin, 1996: Linearization of a longwave radiation scheme for intermediate tropical atmospheric models. $J$. Geophys. Res., 101, 15 129-15 145.

$\longrightarrow$ - - , and H. Su, 2000: Ocean-atmosphere-land feedbacks in an idealized monsoon. Quart. J. Roy. Meteor. Soc., 126, 1-29.

Clement, A., and R. Seager, 1999: Climate and the tropical oceans. J. Climate, 12, 3383-3401.

Emanuel, K. A., J. D. Neelin, and C. S. Bretherton, 1994: On largescale circulations in convecting atmospheres. Quart. J. Roy. Meteor. Soc., 120, 1111-1143.

Gent, P. R., and J. C. McWilliams, 1983a: The equatorial waves of balanced models. J. Phys. Oceanogr., 13, 1179-1191.

_ , and _ 1983b: Consistent balanced models in bounded and periodic domains. Dyn. Atmos. Oceans, 7, 67-93.

_ , and — $1983 \mathrm{c}$ : Regimes of validity for balanced models. Dyn. Atmos. Oceans, 7, 167-183.

Gill, A. E., 1980: Some simple solutions for heat-induced tropical circulation. Quart. J. Roy. Meteor. Soc., 106, 447-462.

Held, I. M., and B. J. Hoskins, 1985: Large-scale eddies and the general circulation of the troposphere. Advances in Geophysics, Vol. 28, Academic Press, 3-31.

Jorgensen, D. P., M. A. LeMone, and B. J.-D. Jou, 1991: Precipitation and kinematic structure of an oceanic mesoscale convective system. Part I: Convective line structure. Mon. Wea. Rev., 119, 2608-2637.

Kingsmill, D. E., and R. A. Houze Jr., 1999a: Kinematic characteristics of air flowing into and out of precipitating convection over the west Pacific warm pool: An airborne Doppler radar survey. Quart. J. Roy. Meteor. Soc., 125, 1165-1207.

— and $1999 \mathrm{~b}$ : Thermodynamic characteristics of air flowing into and out of precipitating convection over the west Pacific warm pool. Quart. J. Roy. Meteor. Soc., 125, 1209-1229.

Lin, J. W.-B., J. D. Neelin, and N. Zeng, 2000: Maintenance of tropical intraseasonal variability: Impact of evaporation-wind feedback and midlatitude storms. J. Atmos. Sci., 57, 2793-2823.

Lindzen, R. S., 1967: Planetary waves on beta-planes. Mon. Wea. Rev., 95, 441-451.

Matsuno, T., 1966: Quasi-geostrophic motions in the equatorial area. J. Meteor. Soc. Japan, 44, 25-43.

McWilliams, J. C., 1985: A uniformly valid model spanning the regimes of geostrophic and isotropic, stratified turbulence: Balanced turbulence. J. Atmos. Sci., 42, 1773-1774.

Miller, R. L., 1997: Tropical thermostats and low cloud cover. J. Climate, 10, 409-440.

Murakami, T., and J. Matsumoto, 1994: Summer monsoon over the 
Asian continent and western north Pacific. J. Meteor. Soc. Japan, 72, 719-745.

Neelin, J. D., 1997: Implications of convective quasi-equilibrium for the large-scale flow. The Physics and Parameterization of Mois Atmospheric Convection, R. K. Smith, Ed., Kluwer Academic, 413-446.

— , and I. M. Held, 1987: Modeling tropical convergence based on the moist static energy budget. Mon. Wea. Rev., 115, 3-12.

_ , and J.-Y. Yu, 1994: Modes of tropical variability under convective adjustment and the Madden-Julian oscillation. Part I: Analytical theory. J. Atmos. Sci., 51, 1876-1894.

- , and N. Zeng, 2000: The first quasi-equilibrium tropical circulation model-formulation. J. Atmos. Sci., 57, 1741-1766.

Numaguti, A., O. Riko, K. Nakamura, K. Tsuboki, N. Misawa, T. Asai, and Y.-M. Kodama, 1995: 4-5-day-period variation and low-level dry air observed in the equatorial western Pacific during the TOGA-COARE IOP. J. Meteor. Soc. Japan, 73, 267290.

Parsons, D., and Coauthors, 1994: The integrated sounding system: Description and preliminary observations from TOGA COARE. Bull. Amer. Meteor. Soc., 75, 553-567.

Pierrehumbert, R. T., 1995: Thermostats, radiator fins, and the local runaway greenhouse. J. Atmos. Sci., 52, 1784-1806.

Raymond, D. J., 1992: Nonlinear balance and potential vorticity thinking at large Rossby number. Quart. J. Roy. Meteor. Soc., 118, 987-1015.

_ 1994: Nonlinear balance on an equatorial beta plane. Quart. J. Roy. Meteor. Soc., 120, 215-219.

- 2000: The thermodynamic control of tropical rainfall. Quart. J. Roy. Meteor. Soc., 126, 889-898.

_ 2001: A new model of the Madden-Julian oscillation. J. Atmos. Sci., 58, 2807-2819.

Reed, R. J., A. Hollingsworth, W. A. Heckley, and F. Delsol, 1988a: An evaluation of the performance of the ECMWF operational system in analyzing and forecasting easterly wave disturbances over Africa and the tropical Atlantic. Mon. Wea. Rev., 116, 824 865.

— E. Klinker, and A. Hollingsworth, 1988b: The structure and characteristics of African easterly wave disturbances as determined from the ECMWF operational analysis/forecast system. Meteor. Atmos. Phys., 38, 22-33.

Rennó, N. O., K. A. Emanuel, and P. H. Stone, 1994a: Radiativeconvective model with an explicit hydrologic cycle. 1: Formu- lation and sensitivity to model parameters. J. Geophys. Res., 99, 14 429-14 441.

_ P. H. Stone, and K. A. Emanuel, 1994b: Radiative-convective model with an explicit hydrologic cycle. 2: Sensitivity to large changes in solar forcing. J. Geophys. Res., 99, 17 001-17 020.

Rhines, P. B., 1975: Waves and turbulence on a $\beta$-plane. J. Fluid Mech., 69, 417-443.

Sardeshmukh, P. D., and B. J. Hoskins, 1987: Derivation of the divergent flow from the rotational flow: The $\chi$ problem. Quart. $J$. Roy. Meteor. Soc., 113, 339-360.

— idealized tropical divergence. J. Atmos. Sci., 45, 1228-1251.

Sherwood, S. C., 1999: Convective precursors and predictability in the tropical western Pacific. Mon. Wea. Rev., 127, 2977-2991.

Sobel, A. H., and C. S. Bretherton, 2000: Modeling tropical precipitation in a single column. J. Climate, 13, 4378-4392.

Stevens, D. E., H.-C. Kuo, W. H. Schubert, and P. E. Ciesielski, 1990: Quasi-balanced dynamics in the tropics. J. Atmos. Sci., 47, 22622273.

Xie, S.-P., and N. Saiki, 1999: Abrupt onset and slow seasonal evolution of summer monsoon in an idealized GCM simulation. $J$. Meteor. Soc. Japan, 77, 949-968.

Yoneyama, K., and T. Fujitani, 1995: The behavior of dry westerly air associated with convection observed during TOGA-COARE R/V Natsushima cruise. J. Meteor. Soc. Japan, 73, 291-304.

- and D. B. Parsons, 1999: A proposed mechanism for the intrusion of dry air into the tropical western Pacific region. $J$. Atmos. Sci., 56, 1524-1546.

Yu, J.-Y., and J. D. Neelin, 1994: Modes of tropical variability under convective adjustment and the Madden-Julian oscillation. Part II: Numerical results. J. Atmos. Sci., 51, 1895-1914.

—, and — 1998: Estimating the gross moist stability of the tropical atmosphere. J. Atmos. Sci., 55, 1354-1372.

Zeng, N., 1998: Understanding climate sensitivity to tropical deforestation in a mechanistic model. J. Climate, 11, 1969-1975.

—_ , and J. D. Neelin, 1999: A land-atmosphere interaction theory for the tropical deforestation problem. J. Climate, 12, 857-872.

$\ldots, \ldots$, and C. Chou, 2000: Quasi-equilibrium tropical circulation model-Implementation and simulation. J. Atmos. Sci., 57, $1767-1796$.

Zhu, B., and B. Wang, 1993: The 30-60-day convection seesaw between the tropical Indian and western Pacific Oceans. J. Atmos. Sci., 50, 184-199.

Zipser, E. M., R. J. Meitin, and M. A. LeMone, 1981: Mesoscale motion fields associated with a slowly moving GATE convective band. J. Atmos. Sci., 38, 1725-1750. 